Peter D. Wearden

William J. Federspiel

Scott W. Morley

Meir Rosenberg

Paul D. Bieniek

Laura W. Lund

Burt D. Ochs

\section{Respiratory dialysis with an active-mixing extracorporeal carbon dioxide removal system in a chronic sheep study}

Received: 2 March 2012

Accepted: 5 July 2012

Published online: 25 August 2012

(C) The Author(s) 2012. This article is published with open access at Springerlink.com

\section{P. D. Wearden}

Children's Hospital of Pittsburgh,

McGowan Institute for Regenerative

Medicine, Pittsburgh, USA

\section{W. J. Federspiel}

McGowan Institute for Regenerative Medicine, University of Pittsburgh,

215 McGowan Institute, 3025 East Carson

Street, Pittsburgh, PA 15203, USA

e-mail: federspielwj@upmc.edu

Tel.: +1-412-3839499

S. W. Morley · M. Rosenberg ·

P. D. Bieniek · B. D. Ochs

ALung Technologies, Inc.,

Pittsburgh, USA

L. W. Lund $(\bowtie)$

Swanson School of Engineering,

University of Pittsburgh, ALung

Technologies, Inc., Pittsburgh, USA

e-mail: 1lund@alung.com

\begin{abstract}
Purpose: The objective of this study was to demonstrate the safety and performance of a unique extracorporeal carbon dioxide removal system (Hemolung, ALung Technologies, Pittsburgh, PA) which incorporates active mixing to improve gas exchange efficiency, reduce exposure of blood to the circuit, and provide partial respiratory support at dialysis-like settings. Methods: An animal study was conducted using eight domestic crossbred sheep, 6-18 months of age and 49-115 kg in weight. The sheep were sedated and intubated, and a 15.5-Fr dual lumen catheter was inserted into the right jugular vein. The catheter was connected to the extracorporeal circuit primed with heparinized saline, and flow immediately initiated. The animals were then awakened and encouraged to stand. The animals were supported in a stanchion and monitored around the clock. Anticoagulation was maintained with heparin to achieve an aPTT of 46-70 s. Results: Measurements included blood flow rate through the
\end{abstract}

device, carbon dioxide exchange rate, pump speed and sweep gas flow rate. Safety and biocompatibility measurements included but were not limited to plasma-free hemoglobin, hematocrit, white blood cell count, platelet count and fibrinogen. The Hemolung removed clinically significant amounts of carbon dioxide, more than $50 \mathrm{ml} / \mathrm{min}$, at low blood flows of $350-450 \mathrm{ml} / \mathrm{min}$, with minimal adverse effects.

Conclusions: The results of 8-day trials in awake and standing sheep supported by the Hemolung demonstrated that this device can consistently achieve clinically relevant levels of carbon dioxide removal without failure and without significant risk of adverse reactions.

Keywords Chronic obstructive pulmonary disease (COPD) . Acute respiratory distress syndrome (ARDS) - Extracorporeal membrane oxygenation (ECMO) ·

Extracorporeal life support (ECLS) · Ventilation - Gas exchange

\section{Introduction}

Extracorporeal carbon dioxide removal $\left(\mathrm{ECCO}_{2} \mathrm{R}\right)$, or "partial ECMO", is a form of extracorporeal life support therapy which has received renewed interest as an adjunct or alternative to invasive mechanical ventilatory support for patients suffering from reversible acute hypercapnic respiratory lung failure [1]. A clinical need for alternative respiratory support therapies providing carbon dioxide $\left(\mathrm{CO}_{2}\right)$ removal exists for both patients experiencing acute exacerbation of chronic obstructive pulmonary disorder (COPD) as well as those with acute respiratory distress syndrome. In acute exacerbation of COPD, patients who do not respond effectively to drug therapy exhibit 
increasing respiratory acidosis and hypercapnia, while remaining normoxic due to supplemental oxygen [2]. Despite the advent and effectiveness of non-invasive ventilation, invasive mechanical ventilation is still required by $26 \%$ to $54 \%$ of patients [3, 4]. The prognosis for COPD patients requiring invasive mechanical ventilation for ventilatory failure is poor, with hospital survival ranging from $76 \%$ to as low as $31 \%$ [5-9]. In respiratory distress syndrome, lung protective ventilation using lower tidal volumes may be complicated by significant $\mathrm{CO}_{2}$ retention, which can lead to arrhythmias and severe dyspnea [10].

Advances in gas exchanger, pump and catheter technologies, in conjunction with the recognized utility of lower flow rate venovenous support modes, have reduced the risks associated with extracorporeal life support, thus opening the door for consideration of its use in earlier stages of acute lung failure [11-14]. The Hemolung respiratory assist system (ALung Technologies, Pittsburgh, PA) is a novel artificial lung device which provides extracorporeal $\mathrm{CO}_{2}$ removal with a cannula and at blood flow rates similar to those used in continuous renal replacement therapy. This device was designed to provide partial respiratory support through a single 15.5 -Fr venovenous catheter at blood flow rates of $350-450 \mathrm{ml} / \mathrm{min}$. The system is unique in that it uses active mixing of the blood adjacent to the hollow fiber membranes (HFM) to increase its gas exchange efficiency, allowing a smaller fiber surface area and reduced flow rates. The levels of oxygenation provided are secondary and recognized to be subtherapeutic.

The purpose of the in vivo study presented in this paper was to demonstrate the safety and performance of the Hemolung for extracorporeal $\mathrm{CO}_{2}$ removal in standing, awake sheep over an 8-day period. The design and methodology of this study was developed to support regulatory approval for clinical testing of the Hemolung. The objective in using healthy sheep was both to maximize the consistency in the length of the study, and to allow evaluation of safety independent of risks associated with disease states or mechanical ventilation. Based on its design and performance in vitro, it was hypothesized that the Hemolung could provide consistent $\mathrm{CO}_{2}$ removal exceeding $50 \mathrm{ml} / \mathrm{min}$ at an arterial $\mathrm{CO}_{2}$ partial pressure $\left(\mathrm{PCO}_{2}\right)$ of $45 \mathrm{mmHg}$ and at blood flow rates in the range $350-450 \mathrm{ml} / \mathrm{min}$. It was further hypothesized that this level of $\mathrm{CO}_{2}$ exchange could be provided without failure or clinically significant adverse reaction in healthy awake sheep for an extended period of 8 days.

\section{Materials and methods}

Animal selection, preparation and baseline assessment

This study was approved by the University of Pittsburgh Institutional Animal Care and Use Committee, and the care and handling of the animals were conducted in accordance with the standards set forth in the USDA Animal Welfare Act and in the current guide for the care and use of laboratory animals prepared by the Institute of Laboratory Animal Resources, National Academy of Sciences. Eight Dorset crossbred sheep weighing 49-115 $\mathrm{kg}$ were used for the study. Prior to surgery, the animals were premedicated with atropine $(0.05 \mathrm{mg} / \mathrm{kg}$, intramuscular) followed by initial anesthetic induction with ketamine hydrochloric acid $(30 \mathrm{mg} / \mathrm{kg}$, intramuscular). Endotracheal intubation was achieved under direct visualization. A surgical plane of anesthesia was achieved using isoflurane (1-3\% to effect) followed by the placement of an oral-gastric tube for abdominal decompression. Monitoring lines were placed in the left external jugular vein and carotid artery. Baseline assessment included arterial pressure, heart rate, blood gases, blood chemistry, complete blood count, activated partial thromboplastin time (aPTT), and plasma-free hemoglobin (PfHb).

\section{Description of Hemolung respiratory assist system}

The primary component of the Hemolung system is a cartridge which houses a cylindrical bundle of siloxane and heparin-coated HFM positioned around a spinning core which simultaneously drives blood flow (centrifugally) through the cartridge and $\mathrm{CO}_{2}$ removal from the blood into the oxygen sweep gas flowing under negative pressure through the fiber lumens (Fig. 1b). The total fiber surface area of the gas exchange cartridge is $0.59 \mathrm{~m}^{2}$. The cartridge is magnetically mated to and driven by an integrated control console (Fig. 1a). Device performance is controlled from the console monitor using two parameters: rotational speed of the cartridge core (rpm) and sweep gas flow $(1 / \mathrm{min})$ through the fibers. The system was interfaced with the sheep through a custom dual lumen 15.5-Fr catheter (Fig. 1c) similar to a dialysis catheter, but with lower flow resistance and greater kink resistance. The volume of the circuit is $280 \mathrm{ml}$ and is primed with sterile $0.9 \%$ sodium chloride containing one unit of heparin per milliliter immediately prior to the procedure.

\section{Surgical procedure}

Following placement of monitoring lines and baseline assessments, the animals were rotated to a left lateral decubitus position. The Hemolung catheter was inserted using a percutaneous Seldinger technique [15]. Following guide wire placement and vessel dilation, a heparin bolus $(80 \mathrm{U} / \mathrm{kg}$ ) was administered. The Hemolung catheter was then advanced into the vasculature under the guidance of fluoroscopy. The tip of the catheter was advanced to the junction of the right atrium and the superior vena cava. 
Fig. 1 Hemolung respiratory assist system: a controller with cartridge magnetically mounted; b schematic of integral gas exchange/pump unit; c dual lumen catheter
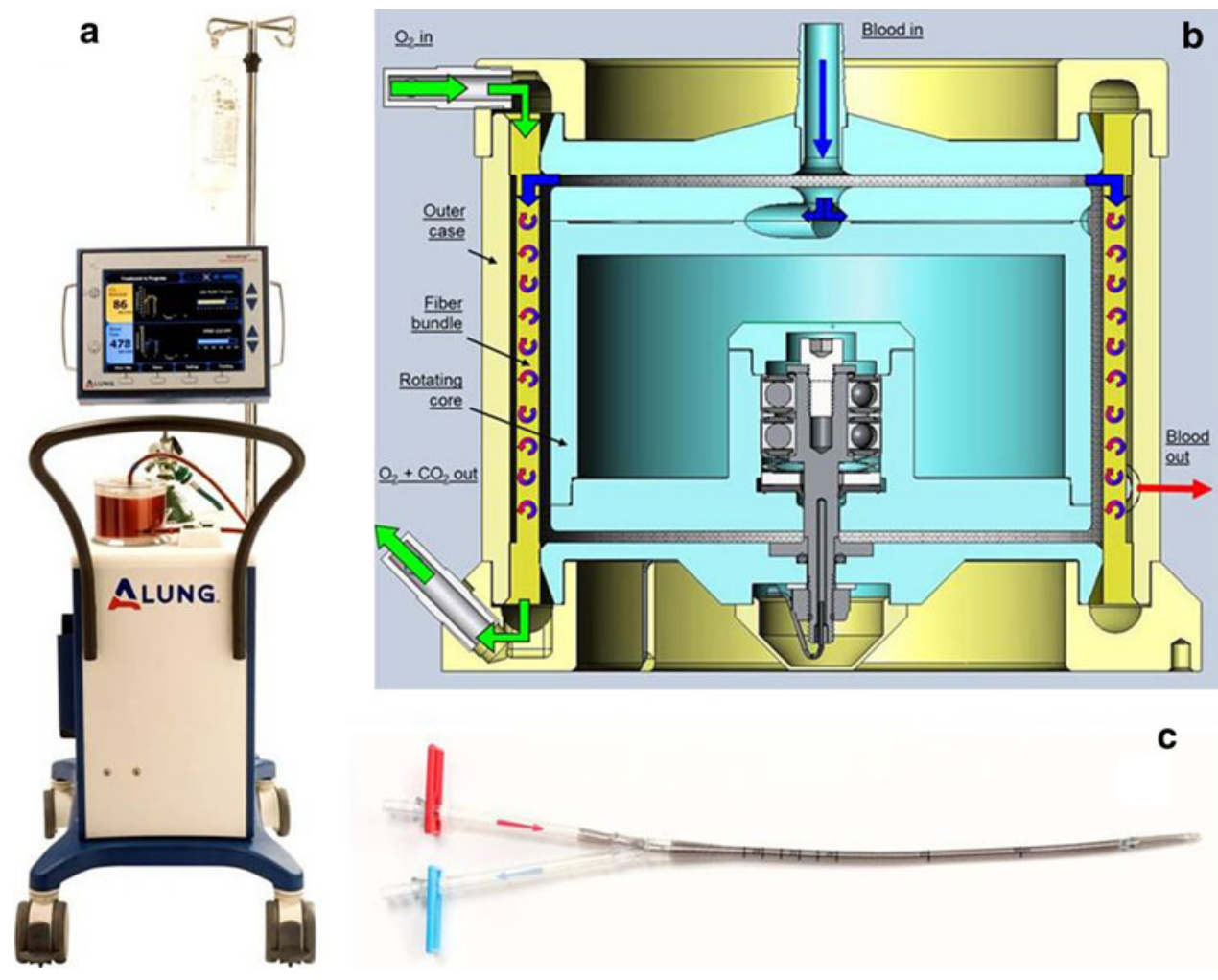

After confirming position, the blood tubing was connected, and extracorporeal flow through the Hemolung circuit immediately initiated. The catheter was secured to the skin with sutures. Hemolung gas flow was set to $8 \mathrm{l} / \mathrm{min}$ and the rotational speed was adjusted to obtain a blood flow of approximately $400 \mathrm{ml} / \mathrm{min}$. A systemic intravenous (IV) heparin drip (100 U heparin per milliliter normal saline) was used to maintain aPTT levels in the range 46-70 s (1.5-2.3 times baseline) during the chronic phase of the trial.

Animal recovery, maintenance, monitoring and measurements

After securing the catheter and initiating Hemolung circulation, anesthesia was discontinued and the animal was extubated. The animal was then transported to the ICU where it was cared for in individual stanchions. Vital signs were monitored hourly over the course of the study, including heart rate, arterial blood pressure, output, and intake. In addition, all animals received prophylactic antibiotic (cephalexin, $3 \mathrm{~g} /$ day IV) and analgesic (flunixin meglumine, $1 \mathrm{mg} / \mathrm{kg}$ IV twice per day for the first 3 days, or longer as needed).

A series of pathology parameters were measured at regular intervals to assess the health of the animal, the performance of the Hemolung device, and the effect of the device on the sheep. These measurements included arterial and venous blood gases, complete blood count, serum chemistry, coagulation parameters, $\mathrm{PfHb}$, urine samples and blood smears.

\section{Hemolung measurements}

The Hemolung cartridge and controller performance was assessed every hour throughout the duration of each trial. Critical measurements included $\mathrm{CO}_{2}$ removal, blood flow, pump speed in revolutions per minute (rpm), and sweep gas flow rate. $\mathrm{CO}_{2}$ removal from the blood into the fiber lumens of the device occurs by diffusion across the wall of the hollow fibers, driven by the difference in $\mathrm{PCO}_{2}$ between the blood and the gas flowing through the fibers. Since variations in blood $\mathrm{PCO}_{2}$ values would result in variable $\mathrm{CO}_{2}$ removal rates, critical evaluation of the $\mathrm{CO}_{2}$ exchange performance required a linear normalization of $\mathrm{CO}_{2}$ removal. The normalized $\mathrm{CO}_{2}$ exchange rate was calculated based on a moving average of venous $\mathrm{PCO}_{2}$, using the following equation:

$$
\mathrm{VCO}_{2}^{\text {norm }}=\mathrm{VCO}_{2}^{\text {actual }} \times \frac{45 \mathrm{mmHg}}{\mathrm{PvCO}_{2}}
$$

Study termination, device removal, and necropsy

After 8 days of data collection the study was terminated. Blood samples were obtained for a complete set of 
terminal blood studies including blood culture. Before the animals were euthanized they were heparinized (100 IU/kg, IV) for anticoagulation. After flushing with heparinized saline, the device was examined for evidence of thrombus deposition. A complete necropsy examination of each animal was performed with the Hemolung catheter in situ. Following explant, the catheter was rinsed with saline and inspected for thrombus and mechanical integrity. The luminal vascular surfaces of the animal were evaluated for potential gross damage related to catheter placement and operation. The organs were evaluated for pathology and evidence of embolic injury. Particular attention was paid to the lungs and the pulmonary arterial vasculature. Representative samples from each of the major organs (lungs, heart, kidneys, liver, and spleen) and the adrenal glands were taken for histopathology.

\section{Results}

Eight healthy sheep were successfully placed on Hemolung therapy under anesthesia, resuscitated, and monitored for up to 8 days. The Hemolung catheter was percutaneously inserted without incident. Recovery from anesthesia was uneventful in all animals. Mechanically, the Hemolung performed as intended with no bearing failures. The Hemolung controller also performed as expected with no loss of sweep gas flow or other failures. Six of the eight animals remained on the device for the entire 8 days. In the second and sixth animals, the Hemolung catheter became dislodged causing premature cessation of treatment. No animals died unexpectedly during the study. The following is a summary of the major findings from the measurements and observations collected prior to, during, and immediately following each animal trial.

Average daily blood flow rates through the Hemolung device are shown in Fig. 2 with corresponding average pump speeds. The normalized $\mathrm{CO}_{2}$ removal rates for each animal are shown in Fig. 3, which includes a table listing the daily average venous $\mathrm{PCO}_{2}$ values. The target blood flow rate of $350-450 \mathrm{ml} / \mathrm{min}$ was obtained at nominal pump speeds. This flow rate successfully supported the target minimum $\mathrm{CO}_{2}$ removal rate of $50 \mathrm{ml} / \mathrm{min}$ at a venous $\mathrm{PCO}_{2}$ of $45 \mathrm{mmHg}$ without degradation in all but one animal. In the fourth animal, which remained on Hemolung therapy for 8 days, a bleeding complication occurred on the third day from a damaged venous monitoring catheter. Blood counts in this animal demonstrated moderate to marked anemia due to bleeding at the site of the venous monitoring line (left neck, opposite side to Hemolung catheter). A blood transfusion was administered on the third day. No other animals experienced bleeding complications.

The results associated with hemocompatibility and organ function are listed in Table 1. The statistical significance of changes over time were evaluated using a

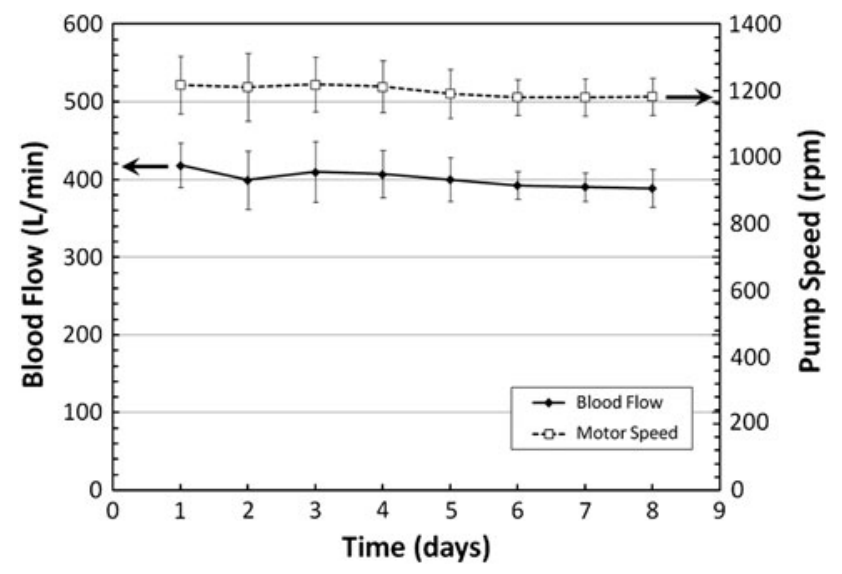

Fig. 2 Average flow rate of blood through the Hemolung circuit (left axis) plotted with average pump motor speed (right axis)

two-tailed heteroscedastic Student's $t$ test. Hemocompatibility measures included hematocrit, platelet count, white blood cell (WBC) count, fibrinogen, PfHb and total bilirubin. Figure 4 shows individual $\mathrm{PfHb}$ measurements for each animal over the course of treatment. Kidney and liver function were assessed using aspartate transaminase (AST), alanine transaminase (ALT), creatinine, and urea nitrogen (BUN). No animal showed any signs of infection and all remained normothermic throughout treatment.

All necropsy and histopathology results were unremarkable, with the exception of the animal which experienced the bleeding complication. There were no gross anatomic lesions found in any of the sheep. All visceral organs were considered to be within normal limits. No intimal vessel damage or vascular clots were seen. Thrombus at the catheter insertion site was seen in some animals. This was considered a normal finding consistent with central venous catheterization. There was no definitive evidence of thromboembolic injury in any of the organs examined, including the lungs. The pulmonary vasculature in all sheep was within normal limits with no evidence of reactive endothelium or thrombus. At necropsy in the fourth animal which experienced the bleeding complication at the site of the venous monitoring line, a kink in the line at the point where it entered the vein was observed. In this animal, there was evidence of minimal subacute to chronic inflammation in the lung, seen as alveolar proteinosis, type II cell hyperplasia, eosinophilic infiltration, and pigmented macrophages. These findings were considered to be a result of the blood transfusion.

Following explant, thrombi of various sizes were found entrained in the bottom gap below the rotating core of three of the eight devices. These thrombi were stable and unable to pass through the fiber bundle, which effectively acted as a filter, and did not affect device performance. Varying amounts of adherent thrombus were found on the fiber bundles, primarily at the warp threads of the fiber mat and the potting junctions, and did 
Fig. 3 Normalized $\mathrm{CO}_{2}$ exchange rate achieved by the Hemolung device in each animal over time. The minimum target level is shown as $50 \mathrm{ml} / \mathrm{min}$ at a $\mathrm{PCO}_{2}$ of $45 \mathrm{mmHg}$. Average daily venous $\mathrm{CO}_{2}$ tensions corresponding to average daily normalized $\mathrm{CO}_{2}$ removal rates in each animal are tabulated below the graph

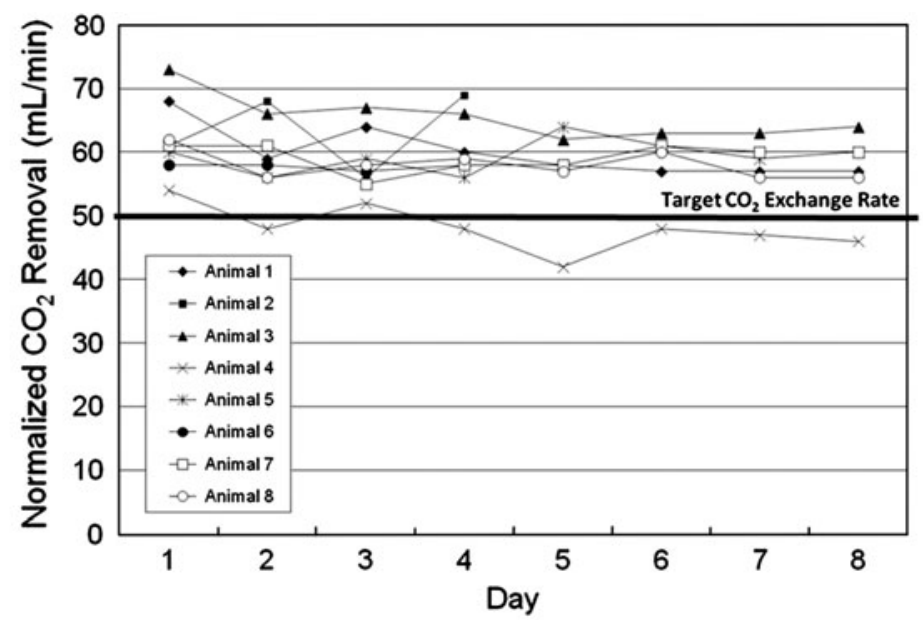

\begin{tabular}{|c|c|c|c|c|c|c|c|c|c|}
\hline & Animal & Day 1 & Day 2 & Day 3 & Day 4 & Day 5 & Day 6 & Day 7 & Day 8 \\
\hline \multirow{8}{*}{$\begin{array}{c}\text { Average } \\
\text { Daily } \\
\text { Venous } \\
\mathrm{PCO}_{2} \\
(\mathrm{mmHg})\end{array}$} & 1 . & $39.5 \pm 3.3$ & $41.0 \pm 2.4$ & $39.4 \pm 3.5$ & $41.6 \pm 2.2$ & $40.3 \pm 0.7$ & $40.1 \pm 1.5$ & $43.0 \pm 5.2$ & $42.1 \pm 3.2$ \\
\hline & 2. & $35.4 \pm 1.9$ & $33.7 \pm 5.7$ & $35.5 \pm 4.4$ & $34.5 \pm 1.3$ & & & & \\
\hline & 34 & $35.7 \pm 1.6$ & $37.0 \pm 1.7$ & $37.6 \pm 2.5$ & $36.1 \pm 1.9$ & $37.6 \pm 1.7$ & $38.2 \pm 3.3$ & $38.1 \pm 1.8$ & $37.3 \pm 2.0$ \\
\hline & $4 \times$ & $39.8 \pm 2.4$ & $42.8 \pm 1.3$ & $45.3^{*}$ & $44.3 \pm 0.6$ & $44.0^{*}$ & $46.0 \pm 1.6$ & $45.7 \pm 3.4$ & $45.5 \pm 0.9$ \\
\hline & $5 *$ & $39.5 \pm 1.5$ & $41.0 \pm 1.4$ & $42.3 \pm 2.4$ & $41.5 \pm 0.6$ & $38.7 \pm 5.5$ & $40.4 \pm 1.1$ & $40.2 \pm 2.1$ & $37.9^{*}$ \\
\hline & $6 \cdot$ & $37.7 \pm 2.4$ & $37.9 \pm 5.0$ & $39.7 \pm 1.5$ & $40.8 \pm 4.6$ & & & & \\
\hline & 70 & $41.0 \pm 4.0$ & $41.8 \pm 2.4$ & $43.9 \pm 1.4$ & $42.5 \pm 3.9$ & $43.0 \pm 3.5$ & $41.0 \pm 5.1$ & $40.7 \pm 2.2$ & $42.2 \pm 0.8$ \\
\hline & 80 & $38.6 \pm 1.1$ & $36.3 \pm 1.5$ & $37.2 \pm 1.5$ & $37.4 \pm 1.0$ & $37.0 \pm 1.5$ & $38.3 \pm 1.2$ & $39.0 \pm 0.9$ & $37.8 \pm 2.0$ \\
\hline
\end{tabular}

not appear to affect gas exchange. The spinning core bearings were all free of any intrusion of blood.

\section{Discussion}

The primary aim of this study was to demonstrate the safety and effective performance of the Hemolung over an 8 -day period. The in vivo model of healthy awake and standing sheep for an 8-day period was used to maximize the ability to evaluate long-term device performance and reliability, and to detect adverse reactions. The primary measurements made to evaluate device performance were the rate of $\mathrm{CO}_{2}$ removal, the rate of blood flow through the device, and the speed of the rotating core of the fiber bundle (other device performance measures were also monitored and recorded). The safety of the therapy provided by the Hemolung was assessed by measurements of animal health, including physiologic monitoring (blood pressure, pulse, and temperature), hematology (complete blood count), serum chemistry, coagulation studies, $\mathrm{PfHb}$, and urinalysis.

The $\mathrm{CO}_{2}$ exchange levels normalized to a venous $\mathrm{PCO}_{2}$ of $45 \mathrm{mmHg}$ were not only consistent over the 8-day period with little degradation (on average, $91 \%$ of initial on day 8), but exceeded the target level by as much as $40 \%$ in all but one animal. In animal four in which $\mathrm{CO}_{2}$ exchange levels were in the range $42-53 \mathrm{ml} / \mathrm{min}$ (normalized), the bleeding complication associated with a damaged venous monitoring catheter resulted in a hematocrit drop of 15-17 \% during the first 4 days of the trial. $\mathrm{CO}_{2}$ transfer in the lung has been shown to decline as a result of reduced hematocrit [16]. In that study, a reduction in hematocrit from $45 \%$ to $15 \%$ was associated with a $50 \%$ reduction in $\mathrm{CO}_{2}$ exchange. This same effect would be expected in the Hemolung and was therefore the likely reason for the reduced $\mathrm{CO}_{2}$ removal rates in this animal.

The target blood flow rate for the Hemolung was $350-450 \mathrm{ml} / \mathrm{min}$, which was consistently observed in all animals. The target blood flow rate was based on the optimal range for achieving clinically significant levels of $\mathrm{CO}_{2}$ removal and on the goal of minimizing exposure of the blood to the extracorporeal system. A fundamental objective in the design process for the Hemolung device was to eliminate the invasiveness, complexity and complications associated with high blood flow ECMO and $\mathrm{ECCO}_{2} \mathrm{R}$ systems used in earlier respiratory assist studies. This aim was achieved through integration of a centrifugal pump within the gas exchanger. The shear and secondary flow patterns set up by the spinning core in the blood adjacent to the surface of the HFMs increases the gas exchange efficiency allowing very low blood flow through the device, as well as simplification of the circuit. A reduced blood flow rate also allowed a reduction in the catheter size needed for percutaneous access to the circulatory system, and therefore the ability to utilize a single venous insertion site with a smaller dual lumen catheter, as opposed to the need for separate cannulations 


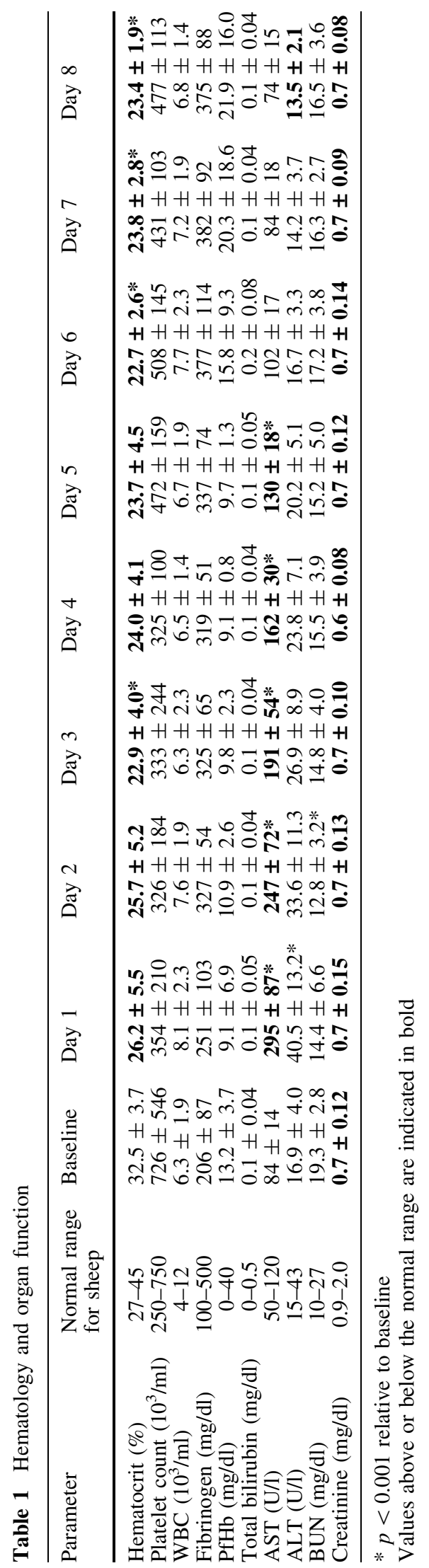

with larger bore catheters required in higher flow applications, which increases the risk of cannulation-associated complications. Furthermore, the intended indications for low flow partial respiratory support excludes patients requiring venoarterial cannulation, which has been shown to be associated with significantly greater mortality [17].

The hematology and organ function measurements made to assess device safety showed changes, but there were no trends or events which indicated significant adverse safety concerns (Table 1). In this study, only one of the eight animals (animal five) exceeded the $40 \mathrm{mg} / \mathrm{dl}$ PfHb threshold for clinically significant hemolysis during the last $48 \mathrm{~h}$ of the study (Fig. 4). At the time of elevated PfHb levels for this animal, total bilirubin remained within the normal range at $0.3 \mathrm{mg} / \mathrm{dl}$ or below, but both hemoglobin and hematocrit fell. Necropsy and explant analysis revealed nothing suggesting that mechanical, device-related hemolysis had occurred in this animal, and pump speed remained stable, indicating no increase in flow resistance through the circuit. Initial increases in the liver function markers AST and ALT were considered to not be indicative of liver damage, but rather a result of muscle damage caused by the cannulation procedure since both enzymes returned to normal over time, and liver necropsy and histopathology results were normal.

The changes seen in hematocrit, in the absence of hemolysis, were likely due to the frequent blood collections for analytic purposes (approximately $40-50 \mathrm{ml}$ of blood per day was drawn for testing) and were not believed to have been caused by the device. Additionally, the hematocrit showed a marked drop perioperatively, even prior to initiation of extracorporeal circulation. It is hypothesized that the perioperative hemodilution due to intravenous fluids, as well as sequestration of red blood cells in the spleen, shifts in body fluids and vasodilation induced by anesthesia, all contributed to this change in hematocrit [18].

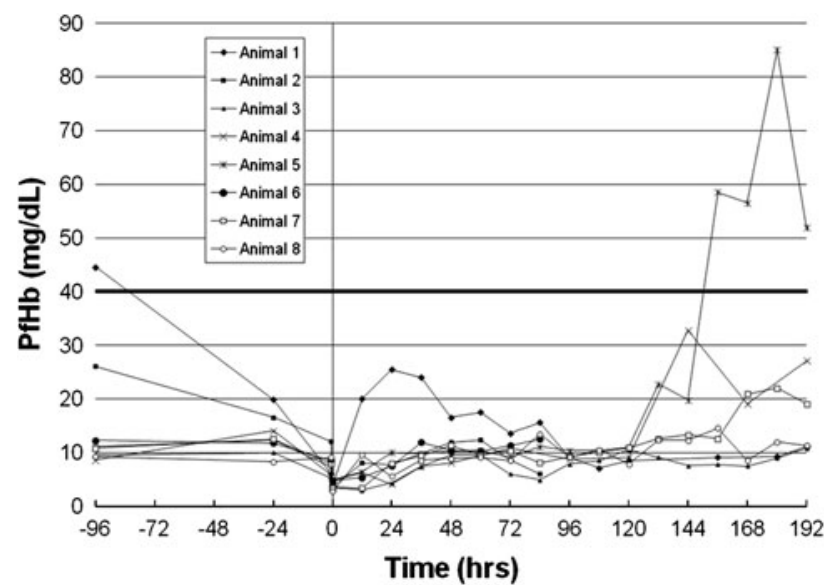

Fig. 4 PfHb over time in each animal. Time zero is immediately following initiation of flow through the Hemolung device while the animal was still under sedation 


\section{Conclusion}

The results of 8-day trials in six awake and standing sheep supported by the Hemolung demonstrate that the Hemolung can consistently achieve clinically relevant levels of $\mathrm{CO}_{2}$ removal without failure and without significant risk of adverse reactions. Normocapnic $\mathrm{CO}_{2}$ exchange rates of $50-70 \mathrm{ml} / \mathrm{min}$ were achieved through a venovenous $15.5-$ Fr dual lumen catheter inserted percutaneously in the right jugular vein at blood flow rates in the range $350-450 \mathrm{ml} / \mathrm{min}$. There were no complications or difficulties experienced with catheter insertion or positioning. The system controller also performed successfully without failure, and was easily monitored and operated by veterinary technicians without substantial respiratory support training. These results support the conclusion that the Hemolung respiratory assist system may be a safe and effective method of $\mathrm{ECCO}_{2} \mathrm{R}$ for partial respiratory support in patients suffering from acute hypercapnic respiratory failure, and that progression to pilot clinical trials in humans can be considered.
Acknowledgments This study was funded by ALung Technologies and was conducted in accordance with regulatory guidelines using an independent principal investigator. In preparation for regulatory review and audit, all possible quality assurance procedures were utilized to validate the integrity of the data.

Conflicts of interest The research described in this article was funded by ALung Technologies, Inc., of Pittsburgh, Pennsylvania. Dr. Federspiel is a founder of ALung Technologies, holds an equity interest in ALung Technologies, and is a paid consultant of the company. Mr. Morley, Dr. Lund and Mr. Bieniek are employees of ALung. Dr. Federspiel, Mr. Morley, Mr. Bieniek, Mr. Ochs and Mr. Rosenberg hold patents and/or have patents pending on the Hemolung technology. Dr. Wearden serves on an independent Data Safety Monitoring Board (DSMB) for ALung Technologies and receives nominal reimbursement for his service in this role.

Open Access This article is distributed under the terms of the Creative Commons Attribution Noncommercial License which permits any noncommercial use, distribution, and reproduction in any medium, provided the original author(s) and the source are credited.

\section{References}

1. Terragni P, Maiolo G, Ranieri VM (2012) Role and potentials of low-flow $\mathrm{CO} 2$ removal system in mechanical ventilation. Curr Opin Crit Care 18:93-98

2. Anon JM, Garcia de Lorenzo A, Zarazaga A, Gomez-Tello V, Garrido G (1999) Mechanical ventilation of patients on long-term oxygen therapy with acute exacerbations of chronic obstructive pulmonary disease: prognosis and cost-utility analysis. Intensive Care Med 25:452-457

3. Quinnell TG, Pilsworth S, Shneerson JM, Smith IE (2006) Prolonged invasive ventilation following acute ventilatory failure in COPD: weaning results, survival, and the role of noninvasive ventilation. Chest 129:133-139

4. Hoo GW, Hakimian N, Santiago SM (2000) Hypercapnic respiratory failure in COPD patients: response to therapy. Chest 117:169-177

5. Stauffer JL, Fayter NA, Graves B, Cromb M, Lynch JC, Goebel P (1993) Survival following mechanical ventilation for acute respiratory failure in adult men. Chest 104:1222-1229

6. Spicher JE, White DP (1987) Outcome and function following prolonged mechanical ventilation. Arch Intern Med 147:421-425
7. Schonhofer B, Euteneuer S, Nava S, Suchi S, Kohler D (2002) Survival of mechanically ventilated patients admitted to a specialised weaning centre. Intensive Care Med 28:908-916

8. Menzies R, Gibbons W, Goldberg P (1989) Determinants of weaning and survival among patients with COPD who require mechanical ventilation for acute respiratory failure. Chest 95:398-405

9. Nava S, Rubini F, Zanotti E, Ambrosino N, Bruschi C, Vitacca M, Fracchia C, Rampulla C (1994) Survival and prediction of successful ventilator weaning in COPD patients requiring mechanical ventilation for more than 21 days. Eur Respir J 7:1645-1652

10. The Acute Respiratory Distress Syndrome Network (2000) Ventilation with lower tidal volumes as compared with traditional tidal volumes for acute lung injury and the acute respiratory distress syndrome. N Engl J Med 342:1301-1308

11. Gattinoni L, Carlesso E, Langer T (2011) Clinical review: extracorporeal membrane oxygenation. Crit Care $15: 243$

12. Terragni PP, Birocco A, Faggiano C, Ranieri VM (2010) Extracorporeal CO2 removal. Contrib Nephrol 165:185-196
13. Terragni PP, Del Sorbo L, Mascia L, Urbino R, Martin EL, Birocco A, Faggiano C, Quintel M, Gattinoni L, Ranieri VM (2009) Tidal volume lower than $6 \mathrm{~mL} / \mathrm{kg}$ enhances lung protection: role of extracorporeal carbon dioxide removal. Anesthesiology 111:826-835

14. Gattinoni L, Carlesso E, Langer T (2012) Towards ultraprotective mechanical ventilation. Curr Opin Anaesthesiol 25(2):141-147

15. Conahan TJ 3rd, Schwartz AJ, Geer RT (1977) Percutaneous catheter introduction: the Seldinger technique. JAMA 237:446-447

16. Bidani A, Crandall ED (1982) Analysis of the effects of hematocrit on pulmonary CO2 transfer. J Appl Physiol Respir Environ Exerc Physiol 53:413-418

17. Brogan TV, Thiagarajan RR, Rycus PT, Bartlett RH, Bratton SL (2009) Extracorporeal membrane oxygenation in adults with severe respiratory failure: a multi-center database. Intensive Care Med 35:2105-2114

18. Hikasa Y, Saito K, Takase K, Ogasawara S (2000) Clinical, cardiopulmonary, hematological and serum biochemical effects of sevoflurane and isoflurane anesthesia in oxygen under spontaneous breathing in sheep. Small ruminant research. J Int Goat Assoc 36:241-249 